\title{
Assuming the worst
}

Daksha Trivedi discusses how a family history of cancer affected her during her diagnosis, and how health professionals can support patients from different cultural backgrounds

\author{
Daksha Trivedi senior research fellow \\ Centre for Research in Public Health and Community Care, University of Hertfordshire, UK
}

The last thing my family had expected to find out and when my mother came to spend Christmas with us was that I had cancer of the lower oesophagus. We were especially in shock and disbelief because only six months previously my twin brother had died of advanced stomach cancer. It felt like lightning had struck twice, and I was taken back to the journey with my brother a few months earlier, which made me fearful of going through the same procedures as him and enduring the same pain. I worried that his fate would be also mine. Knowing what might be to come was hard to deal with emotionally, but having this knowledge allowed me to focus on the things I knew I could control and let go of the things I couldn't.

\section{Cultural background matters}

Many South Asian families have preconceived ideas about cancer that come from stories within their own communities-for example, if one person has had a poor prognosis from cancer, it is assumed that any other person diagnosed with cancer will follow the same path. These assumptions are often made without adequate knowledge or information about treatment options and prognosis. The word cancer often carries a stigma, and some people have their own beliefs about why the cancer has occurred-for example, lifestyle and "karmic consequences" are considered contributing factors.

During my treatment, I believed that everyone would assume the worst about my diagnosis, which would give me little hope to focus on. Finding ways of engaging with people from different cultures, who may process information in different ways, is important. Healthcare professionals can better support South Asian families by being mindful that the families might assume the worst when faced with a cancer diagnosis because of stories from within their community. Communicating facts about the treatment and diagnosis sensitively can help demystify false ideas. For me, the most productive consultation was before surgery and I would have welcomed the discussions which took place then sooner. Being open about the uncertainties will also help patients and their families to better understand the diagnosis rather than presuming the worst.

\section{What carers need to know}

My husband had no choice but to find out more from various sources, which increased his stress further. He started searching online for information on the management and treatment of my cancer, particularly what might be expected of him after surgery. He needed someone to talk to about what to expect after my surgery and information on what was expected of family carers. One source that he found helpful was the Oesophageal Patients Association (www.opa.org.uk), where he was able to read about other patients who had gone through what I was about to experience.

My mother received the information from my husband. She was looking for guidance on how to care for me after surgery, in understanding my symptoms, my functional limitations, and my complex needs, particularly around food and drink. Up until this point, her denial of my diagnosis became her coping mechanism.

After an eight hour operation and several weeks in hospital, we were told that although no further treatment would be required at this stage, regular monitoring would be needed. We have got this far as a family by supporting one another through a long and difficult recovery period. I hope that our community will now see that the worst should not always be assumed.

What you need to know

- It is important to consider the cultural background of patients and their families, particularly older people, who may not understand and feel alone

- Family history of a condition may increase the worry and anxiety after a diagnosis

- The communication of facts and evidence around a diagnosis may provide patients with something positive to focus on 


\section{Education into practice}

- What variation in understanding and information needs do you notice in people from different cultural backgrounds who are facing a cancer diagnosis? Are there ways you could explore concerns or give information differently?

- How could you better ensure that the family members are included?

- How might you discuss a new diagnosis and management plan when the family have already had a member affected by cancer?
Competing interests: I have read and understood BMJ policy on declaration of interests and declare the following: none.

Provenance and peer review: Commissioned, based on an idea from the author; not externally peer reviewed.

Published by the BMJ Publishing Group Limited. For permission to use (where not already granted under a licence) please go to http://group.bmj.com/group/rights-licensing/ permissions 